\title{
New approach towards imaging $\lambda$-DNA using scanning tunneling microscopy/spectroscopy (STM/STS)
}

\author{
SHIRSHENDU DEY, SUSHAMA PETHKAR ${ }^{\dagger}$, SUGUNA D ADYANTHAYA ${ }^{\dagger}$, \\ MURALI SASTRY ${ }^{\dagger \dagger}$ and C V DHARMADHIKARI* \\ DST Unit of Nanoscience and Centre of Advanced Studies in Materials Science and Solid State Physics, \\ Department of Physics, University of Pune, Pune 411 007, India \\ ${ }^{\dagger}$ Nanoscience Group, Materials Chemistry Division, National Chemical Laboratory, Pune 411 008, India \\ ${ }^{\dagger \dagger}$ Tata Chemicals Limited, Leela Business Park, Andheri-Kurla Road, Andheri (E), Mumbai 400 059, India
}

\begin{abstract}
A new methodology to anchor $\lambda$-DNA to silanized $n$-Si(111) surface using Langmuir Blodget trough was developed. The $n$-Si (111) was silanized by treating it with low molecular weight octyltrichlorosilane in toluene. Scanning tunneling microscopy (STM) image of $\lambda$-DNA on octyltrichlorosilane deposited Si substrate shows areas exhibiting arrayed structures of $700 \mathrm{~nm}$ length and $40 \mathrm{~nm}$ spacing. Scanning tunneling spectroscopy (STS) at different stages depict a broad distribution of defect states in the bandgap region of $n$ $\mathrm{Si}(111)$ which presumably facilitates tunneling through otherwise insulating DNA layer.
\end{abstract}

Keywords. DNA; scanning tunneling microscopy; Langmuir Blodget technique; silanization.

\section{Introduction}

DNA imaging always remained a focus of research ever since the invention of scanning tunneling microscopy (STM). Besides imaging, the intrinsic conductivity in DNA has recently been realized to be of immense technological importance with the downscaling in integrated technology where DNA has been recognized as potential candidate, both as a template as well as a functional element, primarily due to its accuracy and precision in programming sequence at nanoscale. This has directed extensive experimental attempts to understand the nature of DNA conductivity over the past few years (Endres et al 2004).

Typical two-probe conductivity measurements carried out so far, where DNA molecule supported on an insulating surface acts as a bridge between two metal electrodes, have reported transport behaviour of DNA to be insulating (Brauns et al 1999; Storm et al 2001), semiconducting (Porath et al 2000), ohmic (Fink and Schönenberger 1999; Tran et al 2000; Rakitin et al 2001), and superconducting (Kasumov et al 2001), which is the result of the complexity of DNA molecule and experimental uncertainties (Endres et al 2004). In addition, DNA molecular characteristics, preparation and detection protocols happen to be obvious factors influencing transport mechanism in DNA. The major challenge in incorporating DNA with the existing semiconductor technology lies in its assembly on desired substrate, specifically on silicon so as to exploit transport through DNA molecule. Various approaches, e.g. electrostatic interactions and post-synthetic conjuga-

*Author for correspondence (cvd@physics.unipune.ernet.in) tion (Strother et al 2000; Sastry et al 2001), or on-chip solid phase synthesis (Patole et al 2003), etc of anchoring DNA to different surfaces have successfully been demonstrated. Attachment of DNA to silicon is generally facilitated via suitable molecular interconnect and therefore, chemical functioning of silicon plays a critical role in deciding the organization of DNA assembly.

In this paper, we propose a protocol to anchor $\lambda$-DNA molecule to silanized silicon surface using Langmuir Blodget trough that could facilitate tunneling through DNA. Earlier studies with tunneling microscopy predominantly include single DNA supported on insulator (Fodor et al 1991) or self-assembled DNA molecules on modified gold (Ceres and Barton 2003) and Si surface with native oxide (Lin et al 1997). Our interest in STM analysis of stretched DNA is mainly driven by technical aspect in the application of molecular combing with electron beam lithography as a promising approach towards integration of DNA-based electronics in current IC fabrication processes. Secondly, with STM, both, topographic and electronic information on nanoscale is experimentally accessible. The stretching mechanism is likely to alter local electronic configuration of DNA that would lead to significant impact on its tunneling characteristics as well. Here, we present, imaging of stretched $\lambda$-DNA molecule supported on silanized Si surface and its characterization using STS.

\section{Experimental and results}

Silanization of Si substrate was acquired from the treatment with low molecular weight silane, which served as 
molecular interconnect. In a typical experiment, $1 \mathrm{sq} \mathrm{cm}$ substrates cut from as received $n$-type Si (111) wafer with resistivity of $\sim 10 \Omega \mathrm{cm}$ were ultrasonically cleaned in isopropyl alcohol (IPA) for 5 min prior to silanization. Silanization was performed under nitrogen atmosphere for $10 \mathrm{~min}$ with $1 \mathrm{mM}$ octyltrichlorosilane (C8 silane) in toluene. $\lambda$-DNA was purchased from MBI Fermentas. $30 \mathrm{ng} / \mathrm{ml} \lambda$-DNA solution was prepared in phosphate buffer saline of $\mathrm{pH} 7 \cdot 2$. To stretch DNA, substrate was vertically immersed in $\lambda$-DNA solution at room temperature, incubated for $20 \mathrm{~min}$ to facilitate DNA interaction with functionalized moiety followed by retracting with the help of Langmuir Blodget technique at the speed of $2 \mathrm{~mm} / \mathrm{min}$ (Michalet et al 1997). The structure of LB film with DNA molecules thus formed on the Si surface was schematically shown in figure 1. The imaging and spectroscopy was performed on both bare Si and silanized Si to ensure stability of the molecular interconnect. Selfassembled monolayer (SAM) of C-8 silane gave stable images enabling steady tunneling current across the barrier.

A home-built scanning tunneling microscope based on a fine mechanical-screw-lever assembly with a compact four-quadrant three-dimensional scanner was used for this investigation. The STM tips were made from $0.25 \mathrm{~mm}$ diameter polycrystalline $\mathrm{Pt}-\mathrm{Rh}$ wires by mechanically cutting them at an angle.

The images were processed using commercially available image processing software (SPIP). The STM imaging was carried out in air with optimum values of tunnel current $(0 \cdot 8-0.9 \mathrm{nA})$ and bias voltage $(-2$ to $+2.5 \mathrm{~V})$ in constant current mode. The tunneling spectroscopy was performed by positioning Pt-Rh tip over an isolated DNA strand by disabling the feedback control, and measuring the $I-V$ characteristics. The $I-V$ characteristics were always repeated several times and finally averaged over tens of runs. The $\mathrm{d} I / \mathrm{d} V$ vs $V$ characteristics were obtained by numerical differentiation of measured $I-V$ curves.

The native oxide layer on $n$-type Si (111) substrate was not stripped before silanization because the formation of natural oxide satisfies dangling bonds that apparently reduces

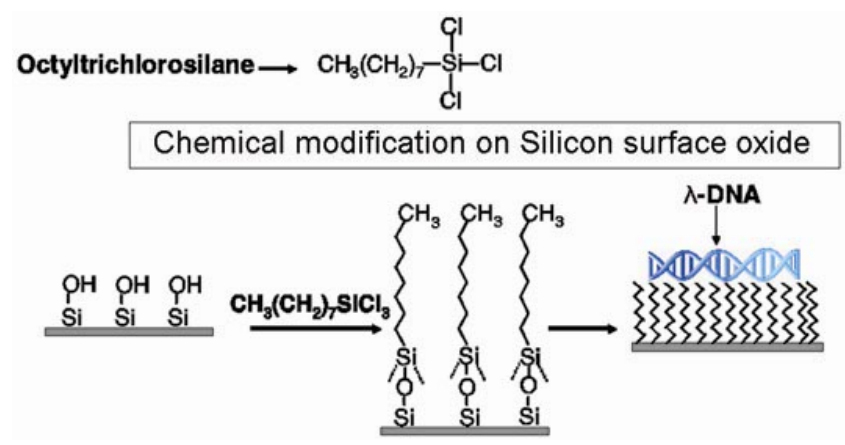

Figure 1. Schematic of LB film structure with attached DNA molecules. the surface state density (Umbach and Blakely 2001). Secondly, it is known that, the oxide surface provides hydroxyl moiety necessary for silanization and native oxide on $\mathrm{Si}$ measures just $\sim 2 \mathrm{~nm}$ thick that enables tunneling of significant electrons for imaging. Figure 2a depicts typical STM image of as received Si substrate with native oxide (termed as bare Si hereafter) obtained with bias voltage of $-2 \mathrm{~V}$ and tunneling current of $0.08 \mathrm{nA}$ at a scan rate of $10 \mathrm{~Hz}$. The STM image of native oxide reveals small-step morphology having a step width of $10 \mathrm{~nm}$ indicating that for the parameters used the tunneling electrons probe $\mathrm{Si}-\mathrm{SiO}_{2}$ interface rather than the surface of the native oxide. The STS curve (figure 3) exhibits asymmetric $I-V$ typical of $n$-type silicon with a bandgap of $\approx 1 \mathrm{eV}$.

As can be seen in figure $2 b$ the add-on layer of $C 8$ silane on bare Si changed the topography dramatically. The thickness of the monolayer as determined from impedance spectroscopy is roughly $1 \cdot 1 \pm 1 \mathrm{~nm}$ with a surface coverage of $98.8 \%$. SAM of alkyltrichlorosilane are found to provide efficient insulation possessing high tunneling energy barriers of $4 \cdot 1-4 \cdot 3 \mathrm{eV}$ for electrons, which has slight dependence on alkyl chain length (Vuillaume et al 1998). The imaging of DNA immobilized on such surface would then be impossible for a reasonable bias potential. The $I-V$ characteristics of silanized bare Si surface must, therefore, be attributed to defect states in the bandgap resulting from disordered molecular layer structure. The presence of defect states is more clear in the conductance curve (figure 4), appearing as a broad peak covering entire bandgap of bare Si. This seems to be advantageous from DNA imaging point of view.

The DNA molecule was stretched on C8 silanized surface. Figure 2c illustrates nearly aligned, well-spaced combed $\lambda$-DNA molecules with typical width of $45 \mathrm{~nm}$ and spacing of $40 \mathrm{~nm}$. It is reasonable to believe that initially electrons from tip tunnel through defect states of SAM into DNA and later from DNA to the tip. Another remarkable observation is the zero conductivity excluding the broad hump originating from silanized surface is recorded up to $3.5 \mathrm{~V}$, which is close to $\pi-\pi^{*}$ gap of DNA $(3.75 \mathrm{eV})$ corresponding to excitation energies of single bases as derived from optical absorption (Endres et al 2004). At room temperature, $\lambda$-DNA is suggested to be an insulator (Brauns et al 1999; Storm et al 2001; Zhang et al 2002) due to static disorder and localized molecular orbitals originating from nonperiodic base pair sequence. The tunneling conductance peak at lower than $3.5 \mathrm{~V}$, bias, therefore, seems to have originated from tunneling of electrons through DNA molecule via defect states of SAM. These preliminary experiments on stretched $\lambda$-DNA spectroscopy support earlier findings (Porath et al 2000), that DNA immobilization protocol influences the electron transport mechanisms in DNA molecule unlike the direct conductivity measurements where the observed gap and the peaks presumably originate from relative position of 

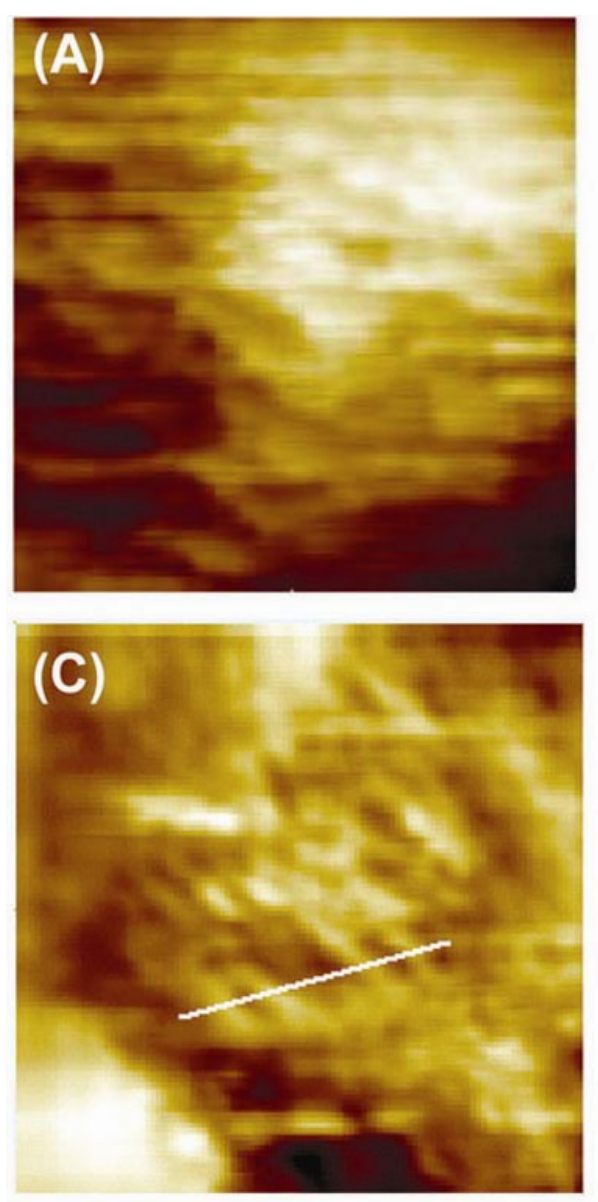

Figure 2. STM images of (A) bare Si [scan size: $100 \times 100 \mathrm{~nm}$ ], (B) silanized Si [scan size: $200 \times 200 \mathrm{~nm}]$, (C) DNA film deposited by Langmuir Blodget technique on the silanized Si substrate [scan size: $1000 \times 1000 \mathrm{~nm}$ ] and $(\mathbf{D})$ line profile along the white line in figure $(\mathbf{C})$.

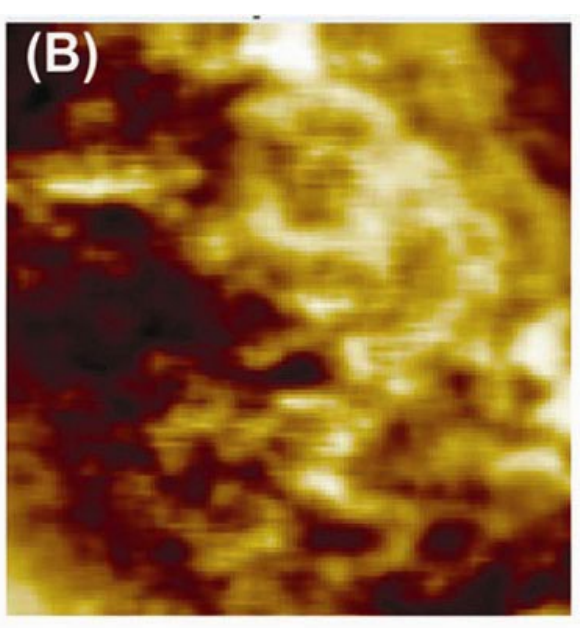

(D)

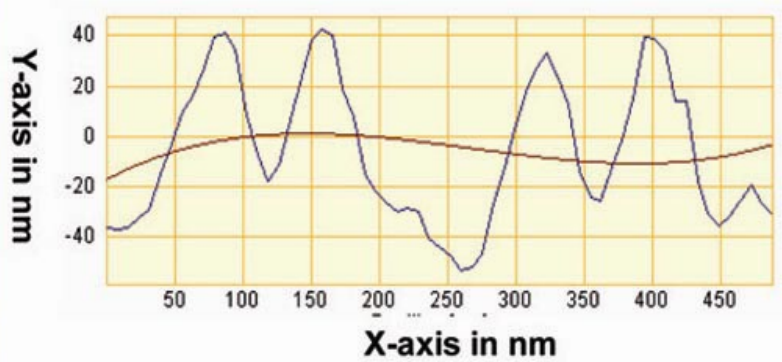

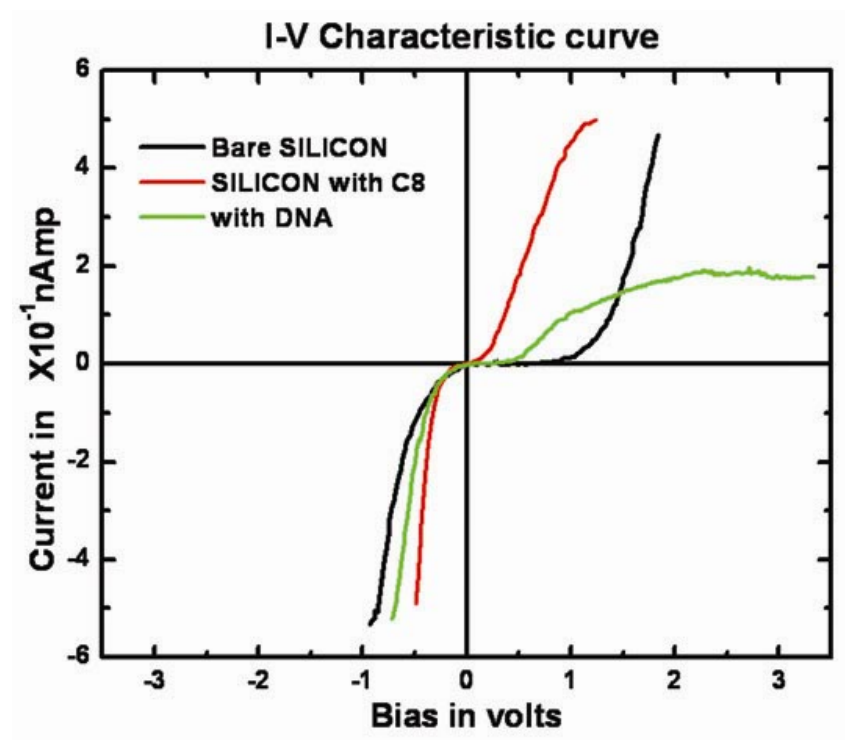

Figure 3. $I-V$ spectra recorded on a bare $\mathrm{Si}$, silanized $\mathrm{Si}$ and on the DNA film deposited by Langmuir Blodget technique on silanized Si substrate. The $I-V$ of the DNA on silanized $\mathrm{Si}$ shows constant current above $2 \cdot 2 \mathrm{~V}$ bias.

$$
\text { . }
$$

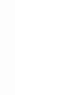


electrode Fermi level and molecular energy bands of DNA and the structure of molecular bands, respectively and in the tunneling transport experiments these parameters seem to be controlled by broad distribution of defect states in disordered octyltrichlorosilane layer. This apparently indicates that functional aspect of DNA in devices could be tailored just by changing fabrication protocols.

\section{Conclusions}

In conclusion, a simple protocol is presented which facilitates STM imaging of DNA molecules on industrially important Si surfaces. STS at different stages depict a broad distribution of defect states in the bandgap region of $n$-Si (111) which facilitate tunneling through otherwise insulating DNA layer. This apparently indicates that the functional aspect of DNA in devices could be tailored by changing fabrication protocols.

\section{Acknowledgements}

One of the authors (SD) would like to acknowledge Pune University-BARC collaborative research program for a research fellowship. (SP) would like to thank the Department of Science and Technology (DST), Government of India, for financial assistance. This work was partially supported by CAS program of UGC, New Delhi, India.

\section{References}

Brauns E B, Madaras M L, Coleman R S, Murphy C J and Berg M A 1999 J. Am. Chem. Soc. 12111644

Ceres D M and Barton J K 2003 J. Am. Chem. Soc. 12514964

Endres R G, Cox D L and Singh R R 2004 Rev. Mod. Phys. 76 195

Fink H W and Schönenberger C 1999 Nature (London) 398407

Fodor S P, Read J L, Pirrung M C, Stryer L, Lu A T and Solas D 1991 Science $\mathbf{2 5 1} 767$

Kasumov A Y, Kociak M, Gueron S, Reulet B, Volkov V T, Klinov D V and Bouchiat H 2001 Science 291280

Lin V S Y, Motesharei K, Dancil K P S, Sailor M J and Ghadiri M R 1997 Science 278840

Michalet X et al 1997 Science 2771518

Patole S N, Pike A R, Connolly B A, Horrocks B R and Houlton A 2003 Langmuir 195457

Porath D, Bezryadin A, Vries S De and Dekker C 2000 Nature (London) $\mathbf{4 0 3} 635$

Rakitin A, Aich P, Papadopoulos C, Kobzar Y, Vedeneev A S, Lee J S and Xu J M 2001 Phys. Rev. Lett. 863670

Sastry M, Kumar A, Datar S, Dharmadhikari C V and Ganesh K N 2001 Appl. Phys. Lett. 782943

Strother T, Hamers R J and Smith L M 2000 Nucleic Acids Res. 283535

Storm A J, Noort J van, Vries S de and Dekker C 2001 Appl. Phys. Lett. 793881

Tran P, Alavi B and Grüner G 2000 Phys. Rev. Lett. 851564

Umbach C C and Blakely J M 2001 Appl. Surf. Sci. 746175

Vuillaume D, Boulas C, Collet J, Allan G and Delerue C 1998 Phys. Rev. B58 16491

Zhang Y, Austin R H, Kraeft J, Cox E C and Ong N P 2002 Phys. Rev. Lett. 89198102 